\title{
Research Paper \\ Comparing the Effects of Rose Essential Oil and Benson Relaxation Technique on Preoperative Anxiety and Hemodynamic Status and Postoperative Com- plications in Rhinoplasty Candidates
}

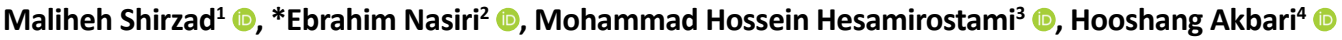

1. Department of Operating Room, Medical Student Research Committee, School of Allied Medical Sciences, Mazandaran University of Medical Sciences, Sari, Iran. 2. Department of Anesthesiology, Operating Room, Traditional and Complementary Medicine Research Center, School of Allied Medical Sciences, Addiction Institute, Mazandaran University of Medical Sciences, Sari, Iran.

3. Department of Plastic and Reconstructive Surgery, Mazandaran University of Medical Sciences, Sari, Iran.

4. Department of Anesthesiology and Operation Room, Faculty of Allied Medical Sciences, Mazandaran University of Medical Sciences, Sari, Iran.

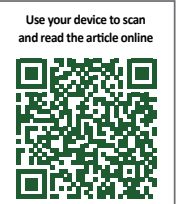

Cittation: Shirzad M, Nasiri E, Hesamirostami MH, Akbari H. [Comparing the Effects of Rose Essential Oil and Benson Relaxation Technique on Preoperative Anxiety and Hemodynamic Status and Postoperative Complications in Rhinoplasty Candidates (Persian)]. Complementary Medicine Journal. 2021; 11(2):180-191. https://doi.org/10.32598/cmja.11.2.1077.1

doi https://doi.org/10.32598/cmja.11.2.1077.1

Key words:

Aromatherapy, Anxiety, Rhinoplasty, Hydrodynamics, Benson relaxation technique

\section{A B STRACT}

Objective Rhinoplasty is a stressful and challenging procedure for plastic surgeries. Researchers are looking for appropriate methods of traditional and complementary medicine to control anxiety before rhinoplasty. This study aims to compare the effects of Benson Relaxation Technique (BRT) and Rose essential oil on preoperative anxiety and hemodynamics and postoperative complications in rhinoplasty candidates. Methods In this randomized intervention study, participants were 68 candidates for rhinoplasty referred to Zare Hospital in Sari, Iran who were randomly divided into BRT $(n=34)$ and aromatherapy $(n=34)$ groups. Anxiety was assessed before and after the intervention by Spielberger's State-Trait Anxiety Inventory. Hemodynamic status, intraoperative bleeding, nausea and vomiting were assessed 24 hours after surgery.

Results The two groups were homogenous in terms of demographic characteristics. The level of overt anxiety decreased by $27.5 \%$ in the aromatherapy group and by $20.4 \%$ in the BRT group. The level of covert anxiety decreased by $27.2 \%$ in the aromatherapy group and by $20.8 \%$ in the BRT group. Systolic and diastolic blood pressure levels decreased more in the aromatherapy group than in the BRT group. There was a significant difference in the number of pulses between the two groups after the intervention $(P=0.038)$. The mean amount of intraoperative bleeding was $18.6 \pm 2.4 \mathrm{~mL}$ in the BRT group and $18.2 \pm 3.8 \mathrm{~mL}$ in the aromatherapy group $(P=0.626)$. Nausea, vomiting and surgeon satisfaction levels during the surgery were similar in the two groups

Conclusion Rose essential oil and BRT are recommended for patients before rhinoplasty due to reducing their preoperative anxiety and stabilizing their hemodynamic status.

\section{Extended Abstract}

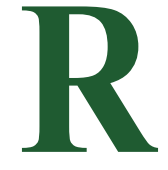

\section{Introduction}

hinoplasty is a stressful and challenging method among plastic surgeries. Anxiety before this surgery is an important problem for patients and the care and treatment team have always tried to reduce or eliminate this problem with appropriate methods. Concern of rhinoplasty candidates about maintaining the beauty of the nasal structure is also very important which can cause their anxiety. According to Shauly et al. [3], about 57.84\%

\section{* Corresponding Author:}

Ebrahim Nasiri, PhD.

Address: Department of Anesthesiology, Operating Room, Traditional and Complementary Medicine Research Center, School of Allied Medical Sciences, Addiction Institute, Mazandaran University of Medical Sciences, Sari, Iran.

Tel: +98 (911) 1517836

E-mail: ebrahimnasiri7836@gmail.com 
of Rhinoplasty candidates suffer from anxiety in the preoperative stage. Anxiety causes a person to respond to a threatening condition and its increase causes physiological changes and the secretion of catecholamines which causes adverse changes in the person's heart, arteries and hemodynamics. Optimal control of anxiety before surgery helps maintain hemodynamic stability and appropriate conditions during surgery and recovery and faster discharge from the hospital. There are various pharmacological and non-pharmacological methods for controlling preoperative anxiety, but various complications such as fatigue, dizziness, restlessness, and respiratory and cardiovascular complications have been reported. Iranian traditional medicine sources consider mental stability as one of the six principles of health and relaxation of body and soul, and suggest medicinal plants to be suitable and useful for creating mental stability and relaxation.. This study aims to compare the effect of rose essential oil and Benson Relaxation Technique (BRT) on anxiety and hemodynamics before rhinoplasty.

\section{Materials and Methods}

In this interventional study, participants were 68 patients who were candidates for rhinoplasty referred to Zare Hospital in Sari, Iran. After obtaining their consent, they were randomly divided into two groups of Aromatherapy $(n=34)$ and BRT $(n=34)$. Inclusion criteria were: age $18-40$ years, ability to understand Persian language, hospitalization at least 4 hours before the intervention, having consent, no hearing or vision problems, no muscle paralysis, and no history of relaxation therapy. Exclusion criteria were sensitivity to rose essential oil, eczema, and having any unpleasant side effects. The sample size was determined 34 for each group with 95\% confidence interval and considering beta error of $20 \%$ and a sample drop of $10 \%$ based on a similar study. Demographic and clinical characteristics and anxiety in both groups were recorded two times, 3-5 hours and 30-60 minutes before surgery. Hemodynamic status was measured before and after the intervention using a digital sphygmomanometer. Anxiety before and after the intervention was assessed by Spielberger's State-Trait Anxiety Inventory. Intraoperative bleeding, agitation in recovery, nausea and vomiting were also assessed 24 hours after surgery. T-test or Mann-Whitney U test were used for analyzing quantitative variables and Chi-square test was used for qualitative variables.

\section{Results}

The results showed no significant difference between the two groups in terms of gender, age, educational level, and occupation. Systolic and diastolic blood pressure decreased more in the aromatherapy group than in the BRT group. The number of pulses between the two groups after intervention was significantly different $(\mathrm{P}=0.038)$. The Mean $\pm \mathrm{SD}$ rate of intraoperative bleeding was $18.6 \pm 2.4 \mathrm{ml}$ in the BRT group and $18.2 \pm 3.8 \mathrm{ml}$ in the aromatherapy group $(\mathrm{P}=0.626)$. Nausea and vomiting and surgeon satisfaction during the surgery were similar in the two groups. The level of overt and covert anxiety in both groups was significantly reduced compared to pre-intervention scores where the level of reduction was higher in the aromatherapy group (Table 1). The mean score of overt anxiety in the pre- and post-intervention stages decreased by $8.9(20.4 \%)$ in the BRT group

Table 1. Comparison of mean scores and changes of overt and covert anxiety before and after intervention in the two groups

\begin{tabular}{|c|c|c|c|c|}
\hline \multirow{2}{*}{ Variable } & \multirow{2}{*}{ Assessment Phases } & \multicolumn{2}{|c|}{ Mean $\pm S D$} & \multirow{2}{*}{ Sig.* } \\
\hline & & BRT Group & Aromatherapy Group & \\
\hline \multirow[b]{2}{*}{ Overt anxiety } & Pre-intervention & $43.7 \pm 8$ & $48.4 \pm 5.9$ & 0.051 \\
\hline & Post-intervention & $34.8 \pm 5.4$ & $35.1 \pm 4.7$ & 0.996 \\
\hline \multicolumn{2}{|c|}{ Sig.* } & 0.001 & 0.001 & \\
\hline \multicolumn{2}{|c|}{ Reduction (Percentage) } & $8.9(20.4)$ & $13.3(27.5)$ & \\
\hline \multirow{2}{*}{ Covert anxiety } & Pre-intervention & $46.7 \pm 6.2$ & $46.7 \pm 6.4$ & 0.996 \\
\hline & Post-intervention & $37 \pm 4.8$ & $34 \pm 5.1$ & 0.047 \\
\hline \multicolumn{2}{|c|}{ Sig.* } & 0.001 & 0.001 & \\
\hline \multicolumn{2}{|c|}{ Reduction (Percentage) } & $8.20(7.9)$ & $12.7(27.2)$ & \\
\hline
\end{tabular}

*Paired t-test. 
and by $13.3(27.5 \%)$ in the aromatherapy group $(\mathrm{P}<0.001)$. Paired $\mathrm{T}$ test results showed significant differences before and after intervention in both groups $(\mathrm{P}<0.001)$. The mean score of covert anxiety in the pre- and post-intervention stages decreased by $9.7(20.8 \%)$ in the BRT group and by $12.7(27.2 \%)$ in the aromatherapy group. Paired T test results showed that this difference before and after intervention in both groups was significant $(\mathrm{P}<0.001)$.

\section{Conclusion}

Due to significant effectiveness of rose essential oil and BRT in reducing preoperative anxiety and maintaining the hemodynamic status of Rhinoplasty candidates, their use is recommended.

\section{Ethical Considerations}

Compliance with ethical guidelines

This study obtained its ethical approval form the ethics committee of Mazandaran University of Medical Sciences (Code: IR.MAZUMS.REC.1398.1445).

Funding

This study was extracted from the MSc thesis of first author at the Department of Operating Room, School of Allied Medical Sciences, Medical Student Research Committee, Mazandaran University of Medical Sciences, Sari.

Authors' contributions

Conceptualization: Ebrahim Nasiri and Mohammadhossein Hesamirostami; methodology: Maliheh Shirzad and Mohammadhossein Hesamirostami; data analysis: Ebrahim Nasiri and Maliheh Shirzad; draft preparation, editing \& review: All authors.

Conflicts of interest

The authors declare no conflict of interest

Acknowledgements

The authors would like to thank the Vice-chancellor for Research of Mazandaran University of Medical Sciences, participants, and personnel of Zare Hospital in Sari for their support and cooperation. 
مقاله يخوهشى

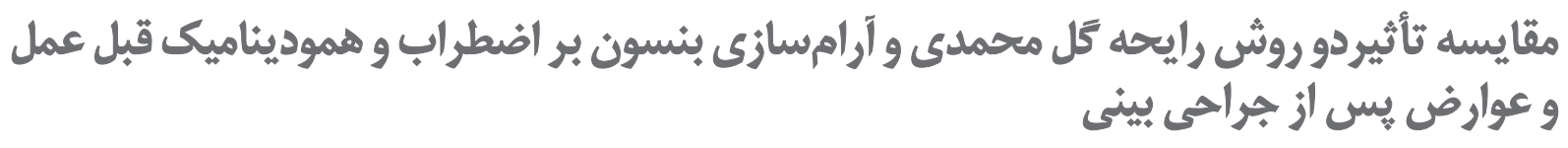

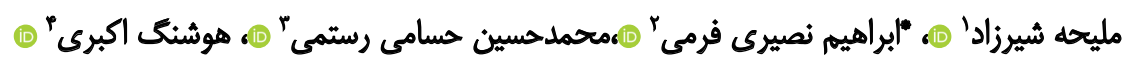

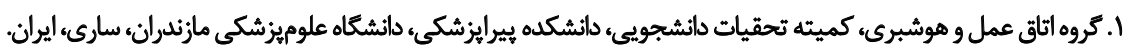

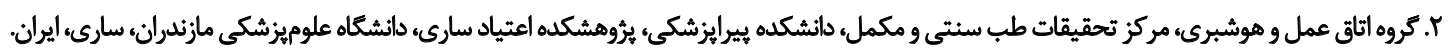

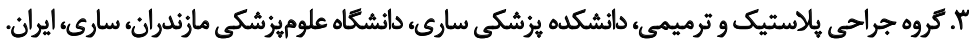

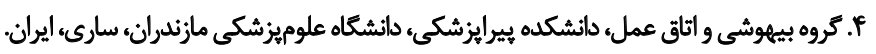

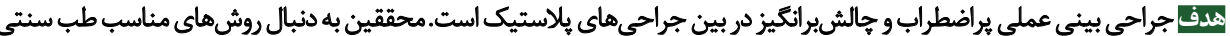

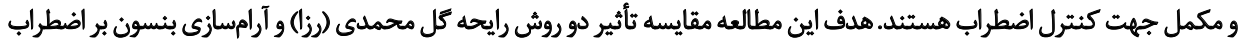

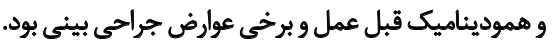

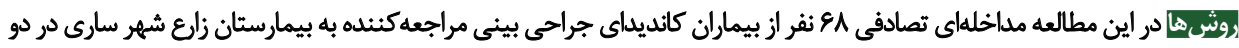

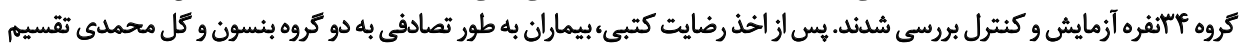

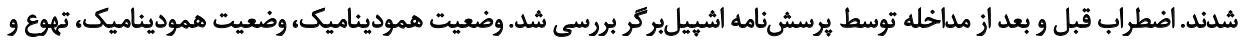

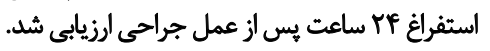

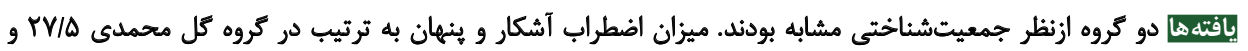

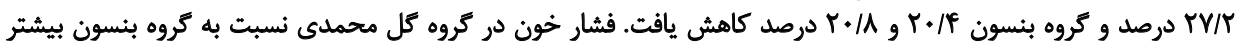

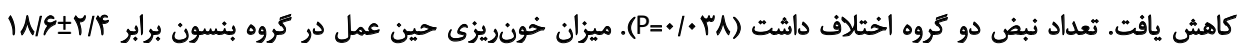

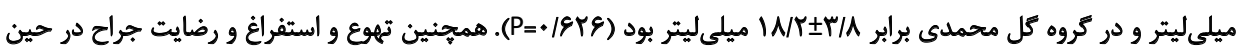

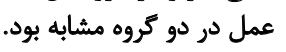

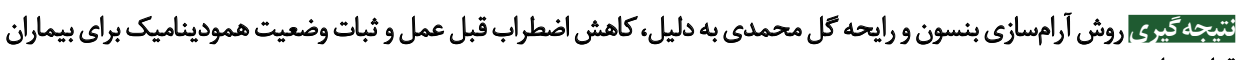
قبل عمل توصيه مى شئود.

كه به روشهاى مناسبى اين مشكل كاهش يابد و از طرف ديكر

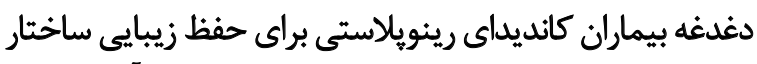

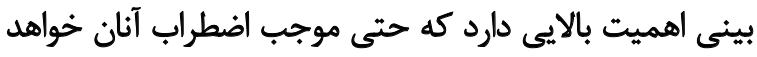

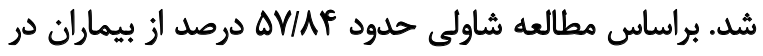

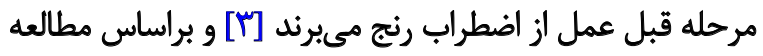

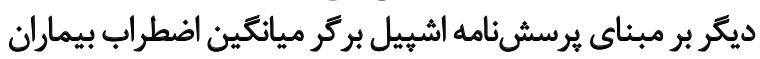

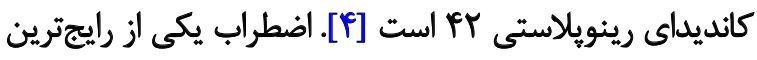

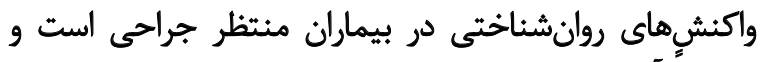

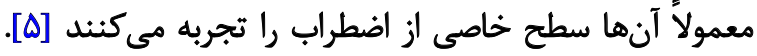

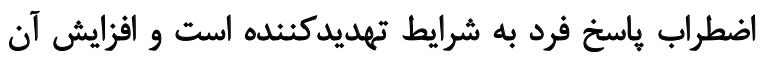

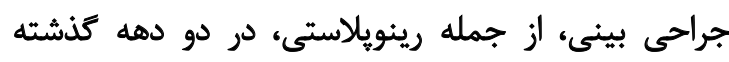

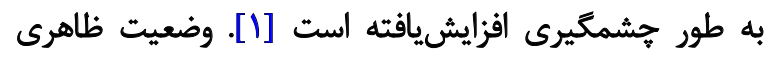

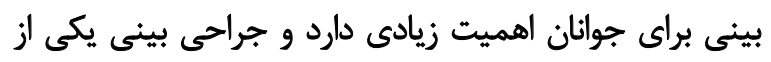

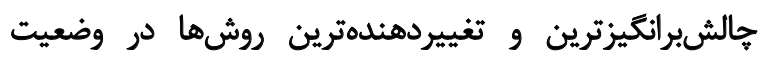

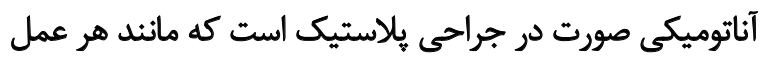

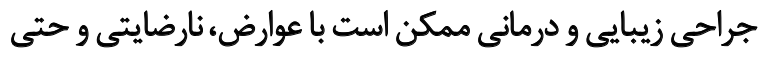

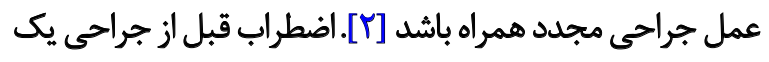

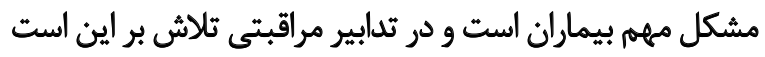

$$
-
$$
نشانى: سارى، دانشعاه علوميزشكى مازئندان، يرؤشككه اعتياد سارى، دانشكده بيرايزشكى، مركز تحقيقات طب سنتى و مكمل، كروه اثاق عمل و هوشبرى. تلفن: يست الكترونيكى: ebrahimnasiri7836@gmail.com 
داده است كه عارضه خاصى در استفاده از رايحه كَل محمدى براى

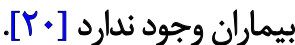

از طرف ديكر آرامسازى بنسون، با ايجاد تعادل بين قسمت

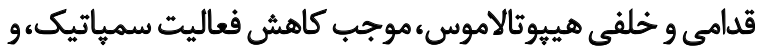

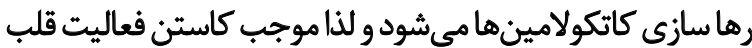

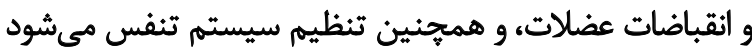

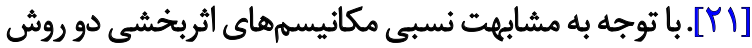

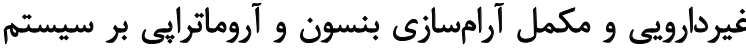

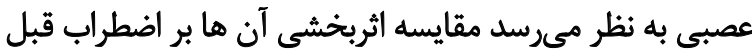
عمل و تغييرات هموديناميكى مفيد باشد.

با توجه به اينكه عمل جراحى بينى از نظر زيبايى و عملكردى ئي

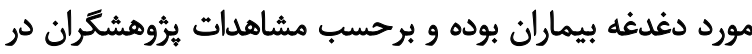

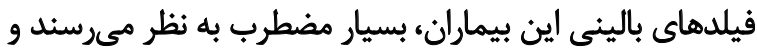

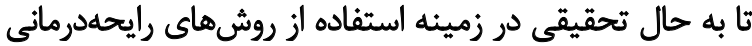

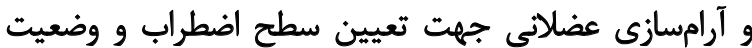

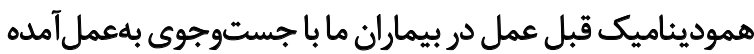

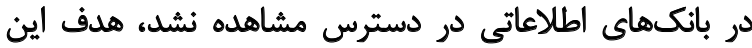

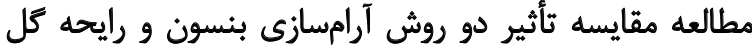

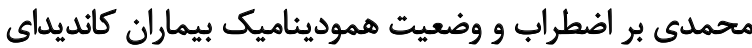
جراحى بينى و عوارض بعد از آن بود.

مواد وروش

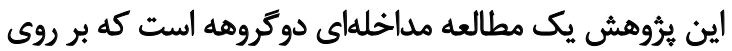

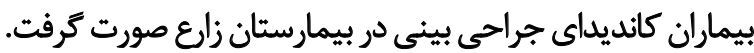

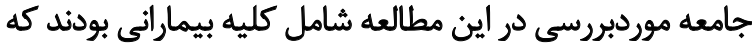

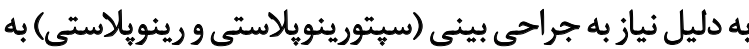
اثاق عمل بيمارستان زايع به صورت مستمر مراجعل كرديند. معيارهاى ورود به اين مطالعه شامل سن 11 - -f سال، توانايى

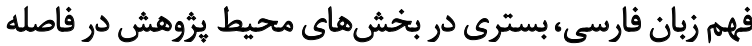

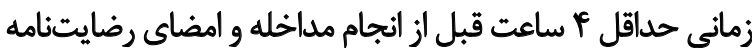

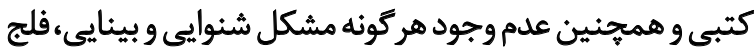

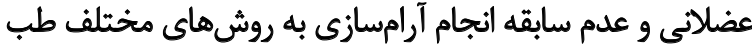

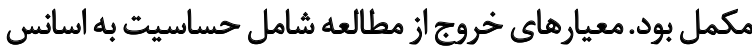

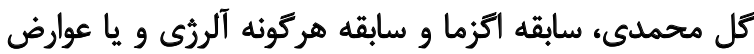
جانبى ايجادشده در طى تحقيق بود.

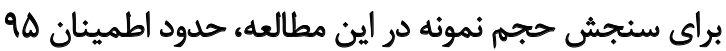

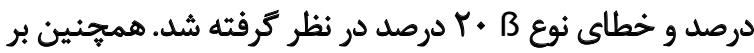

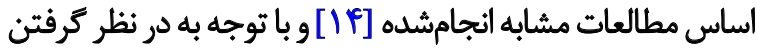

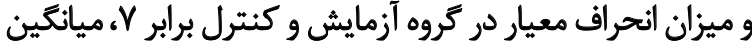

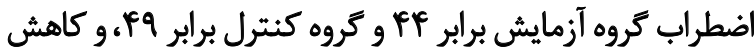

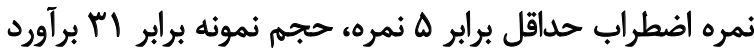

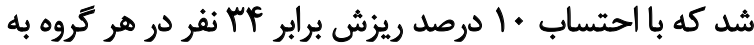

موجب تغييرات فيزيولوزيكي مي شود [ع] كه ممكن است ترشح

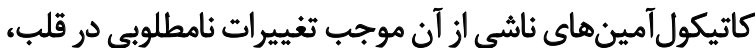

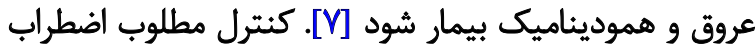

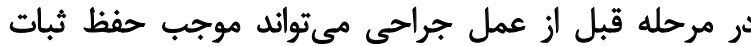

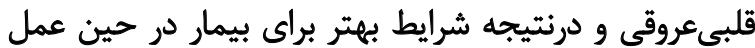

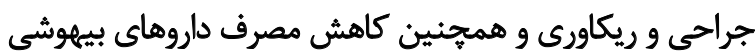

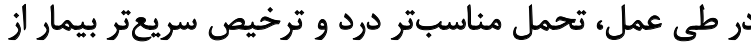

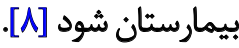
روشهاى دارويى مانئد داروهاى آرامبخش و مخدرها اغلب

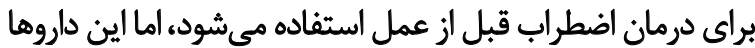

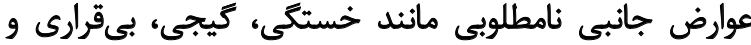

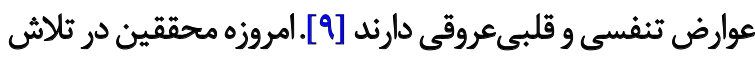

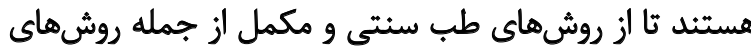

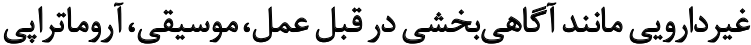

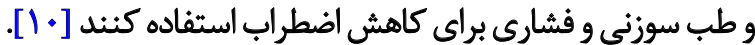

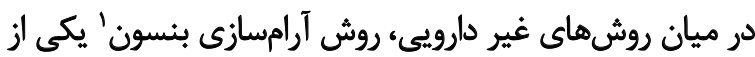

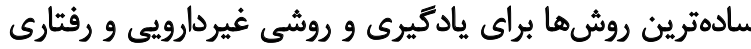

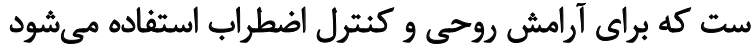

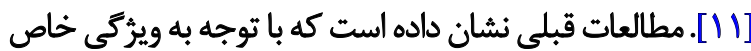
روش بنسون كه عضلات آرامش خاصى بيدان فيدا مي كنيند، كاهش

تعداد نبض، تنفس و ثبات فشار خون حاصل مي خشود [1T]

آروماترايى يكى از متداولترين روشهاى غيردارويى جهت كاهش درد و اضطراب در بيماران است كه در آن آن معمولاًاز اسانس

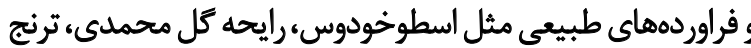

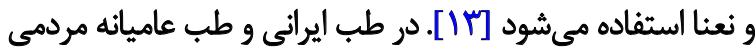

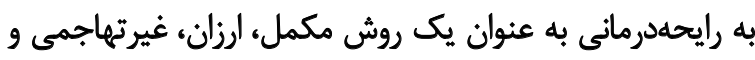

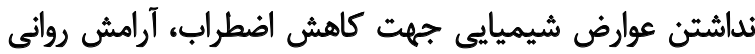

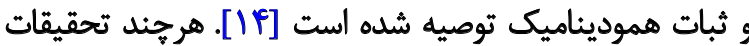

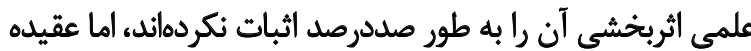

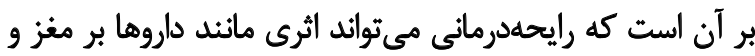

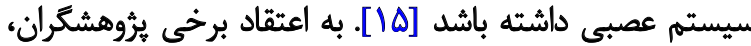

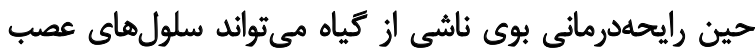

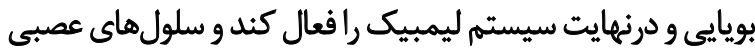

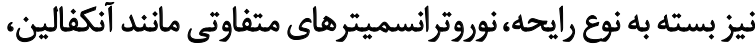

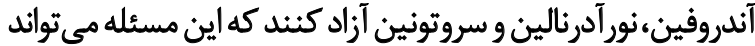

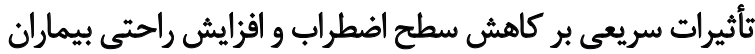

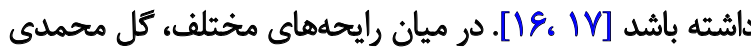

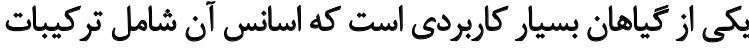

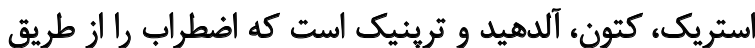

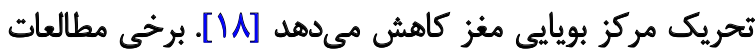

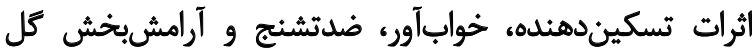

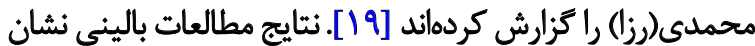

1. Benson Relaxation Method (BRM) 
صورت مصاحبه مستقيم با يرسش از بيمار در تعداد دفعات اين

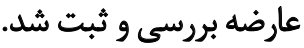

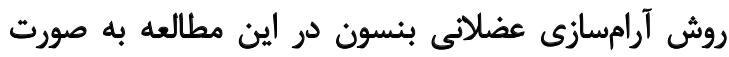

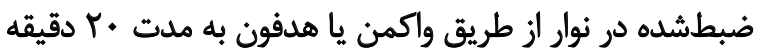

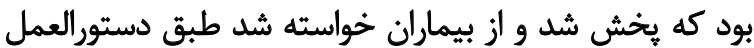

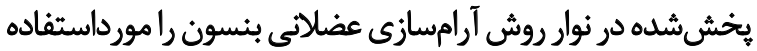

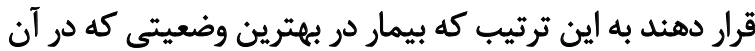

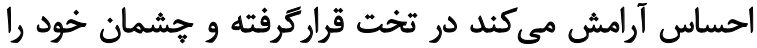

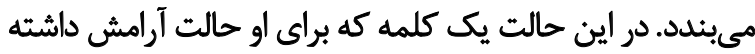

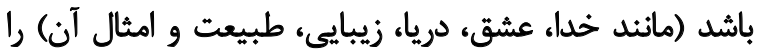

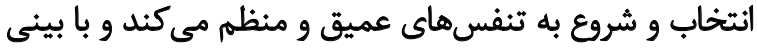

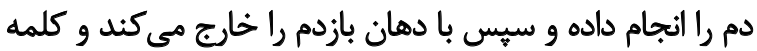

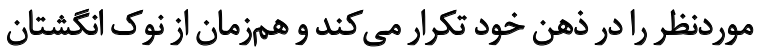

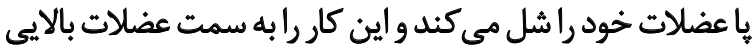

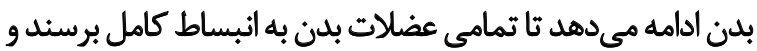

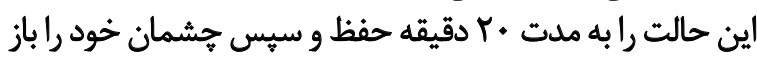

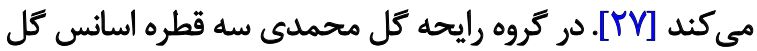

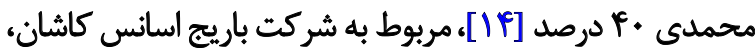

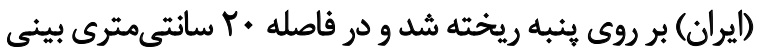

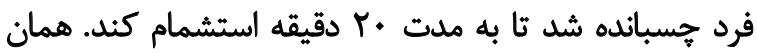

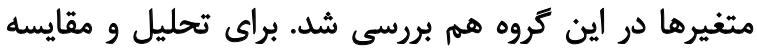

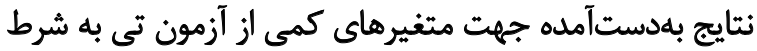

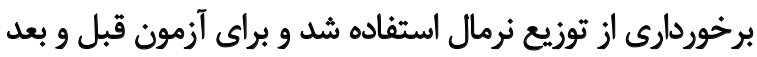

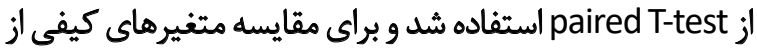

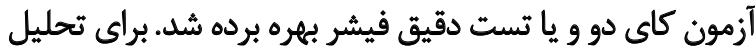

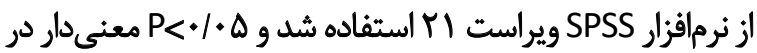

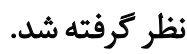

يافتهها

نتايج نشان داد كه ه ع بيمار در دو گروه آانفرى شركت داشتئد

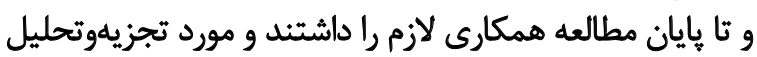
قرار كرفتند (تصوير شماره )). نتايج تحقيق نشان داد كه ميانكين سن بيماران در كروه بنسون

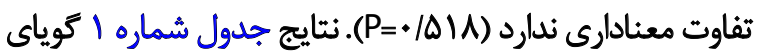

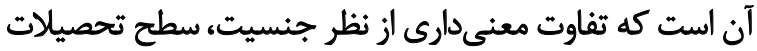

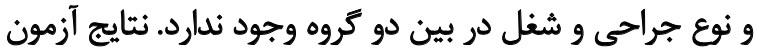

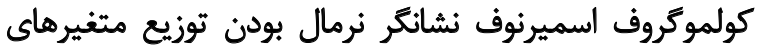
موردبررسى در دو كروه بود.

ميانكين ضربان قلب در مراحل مختلف اندازهكيرى تفاوت

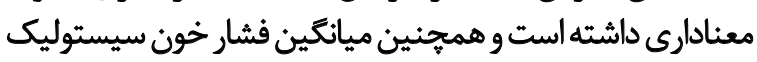

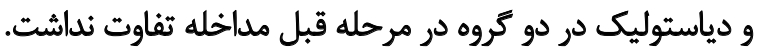

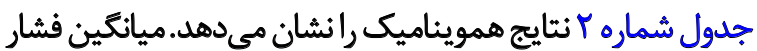

$$
\text { دست آمدكه مورد بررسى قرار گرفت (فرمول شماره ) }
$$

$$
\mathrm{n}=\frac{\left(\mathrm{s}_{1} \mathrm{~d}^{2}{ }_{1}+\mathrm{s}_{2} \mathrm{~d}_{2}{ }_{2}\right)\left(\mathrm{Z}_{1-\frac{\alpha}{2}}+\mathrm{Z}_{1-\beta}\right)^{2}}{\left(\mathrm{M}_{1}-\mathrm{M}_{2}\right)^{2}}
$$

بيماران به طور تصادفى با استفاده از روش - Block Random

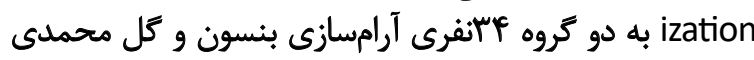

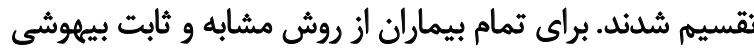

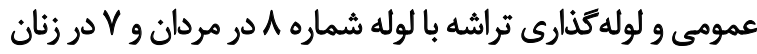

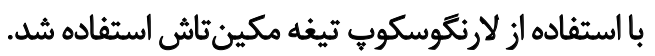

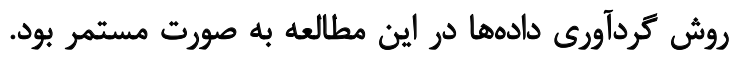

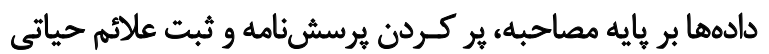

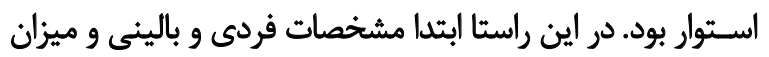

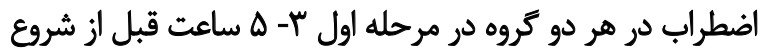

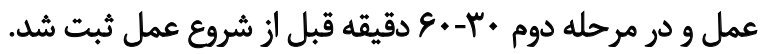

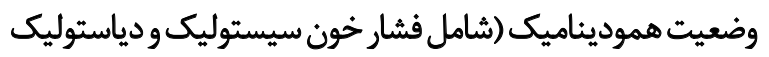

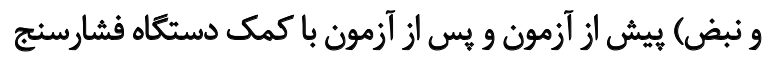

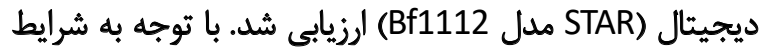

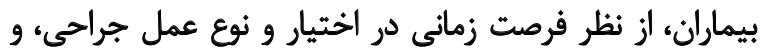

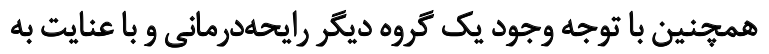

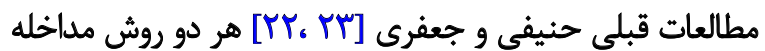

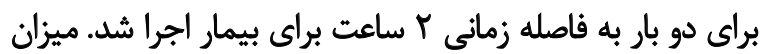

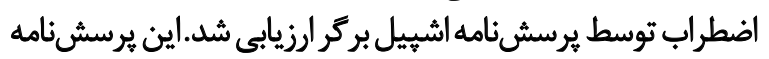

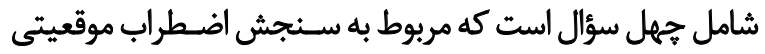
(آشكار و ينهان) است. حداقل امتيازات كسبشال استه (به تفكيك)

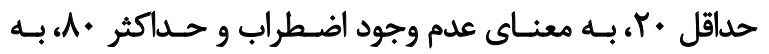

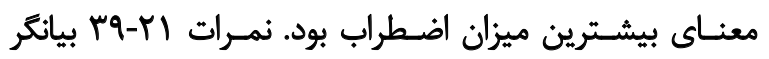

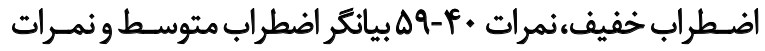

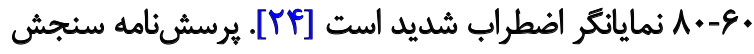

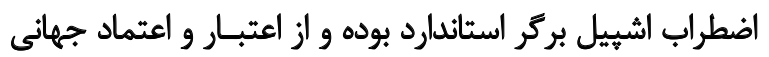

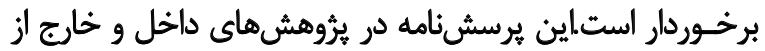

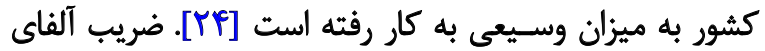

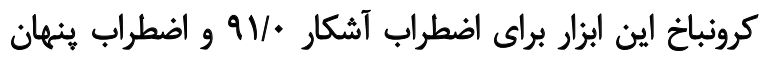

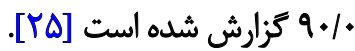

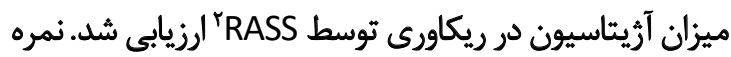

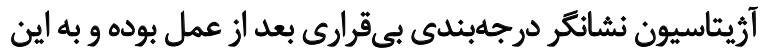

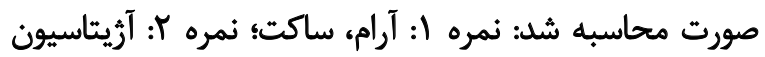

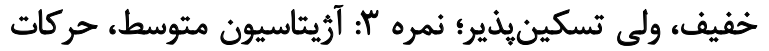

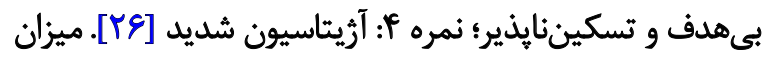

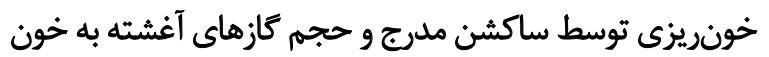

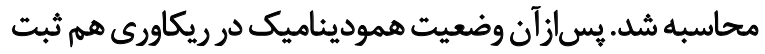

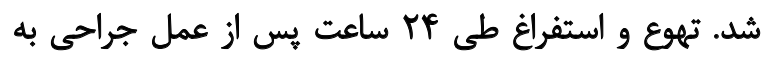

2. Sedation Scale Richmond Agitation 


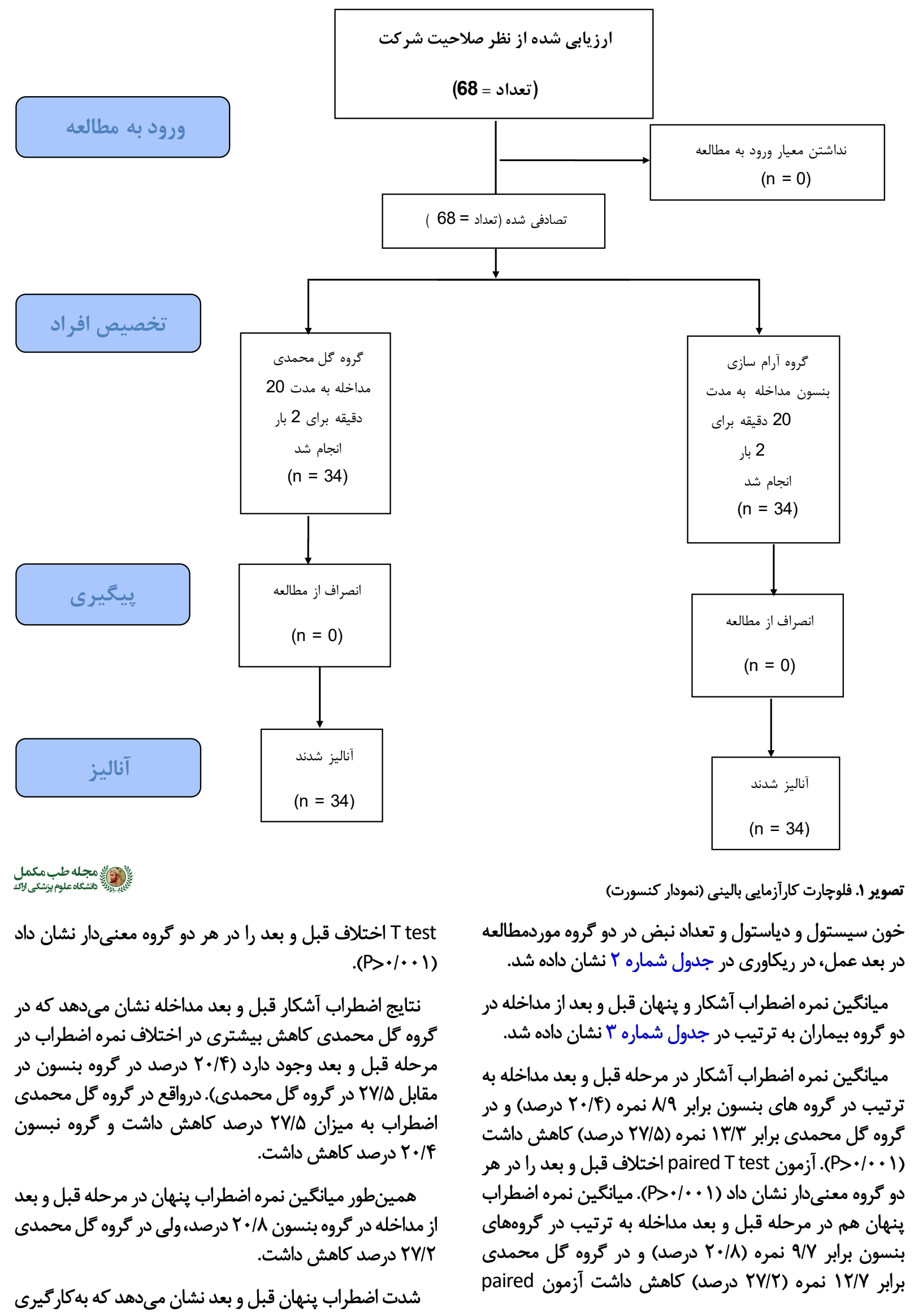

شدت اضطراب ينهان قبل و بعد نشان مى دهد كه بهكاركيرى 
جدول ا. فراوانى متغيرهاى جمعيتشناختى جنس، سطح تحصيلات و غيره به تفكيك در دو كروه

\begin{tabular}{|c|c|c|c|c|}
\hline \multirow{2}{*}{ "سطح معنى دار } & \multicolumn{2}{|c|}{ فراوانى (درصد) } & \multirow{2}{*}{\multicolumn{2}{|c|}{ مثغير ها كروه }} \\
\hline & كل محمدى & بنسون & & \\
\hline \multirow{2}{*}{$\cdot M Q 1$} & $\Pi\left(M T / T^{\circ}\right)$ & $\mid r(r \Delta / r)$ & مرد & \multirow{2}{*}{ 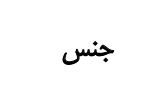 } \\
\hline & $M(e V / \varepsilon)$ & $r r(e+/ V)$ & ن & \\
\hline \multirow{2}{*}{ toEV } & $V(r \cdot / \varphi)$ & $q(\mu / \Delta)$ & دييلم و كمتر & \multirow{2}{*}{ سطح تحصيلات } \\
\hline & $r V(Y Q / F)$ & $r \Delta(Y r / \Delta)$ & بالاتر از دييلم & \\
\hline \multirow{2}{*}{. /ATA } & $Y(Y+/ 8)$ & $\Delta(I F / V)$ & رينويلاستى & \multirow{2}{*}{ نوع جراحى } \\
\hline & $r v(v a / 4)$ & $r q\left(\Lambda \Delta / T^{\prime}\right)$ & سيتورينويلاستى & \\
\hline \multirow{4}{*}{. MET } & $\varepsilon(I V / \&)$ & $V(Y+19)$ & آزاد & \multirow{4}{*}{ شئل } \\
\hline & $M(M T / F)$ & $P(W / A)$ & 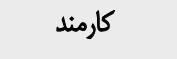 & \\
\hline & $1 \cdot(r q / 4)$ & $1 \cdot(r q / P)$ & كائشجو & \\
\hline & $V(Y \cdot / \varepsilon)$ & $\mathbb{I r}\left(Y_{N} N\right)$ & خائهدار & \\
\hline
\end{tabular}

است كه تفاوت معنى دارى از نظر جنسيت، سن، سطح تحصيلات

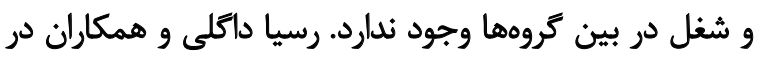

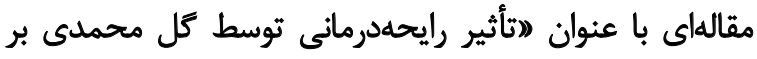

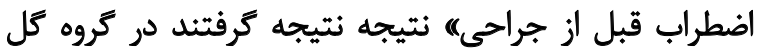

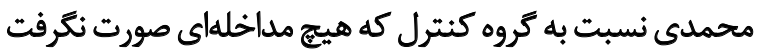

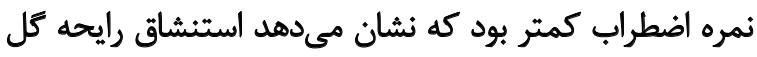

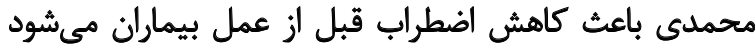

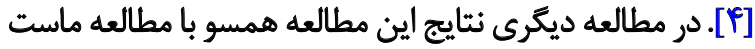

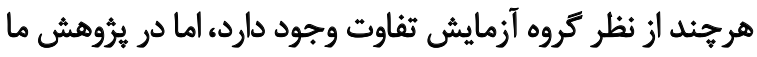

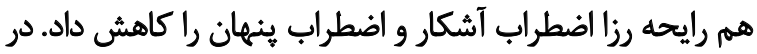

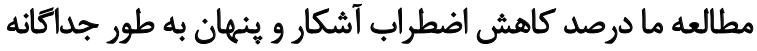

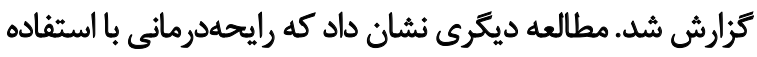

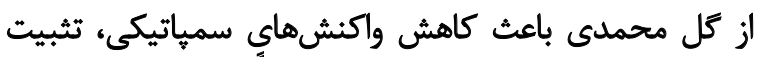

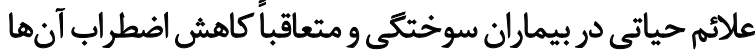

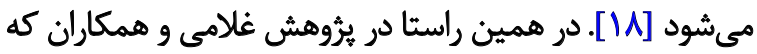

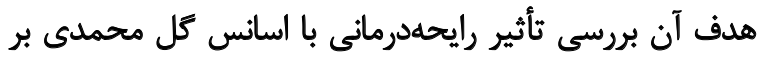

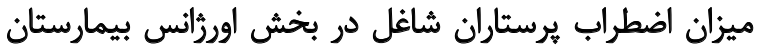

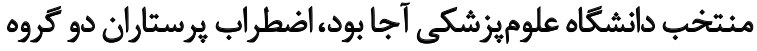

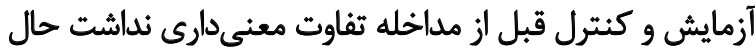

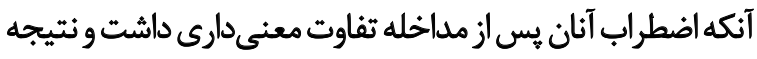

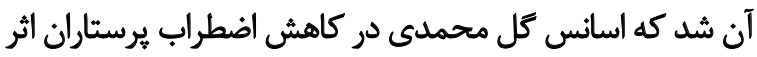

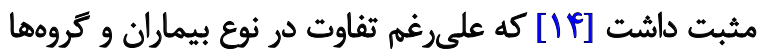
نتيجه همسو با مطالعه ما به دست آمدا در مطالعه جعفرى و همكاران تأثير آرامسازى عضلانى بنسون بر سطح اضطراب بيماران در انتظار مداخلات تشخيص آرانيى و درمانى

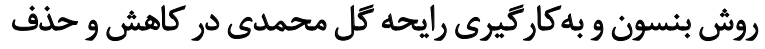

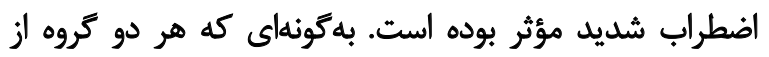

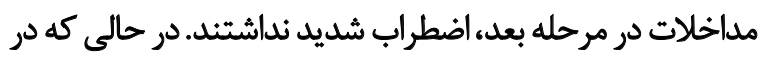

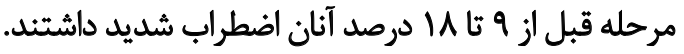

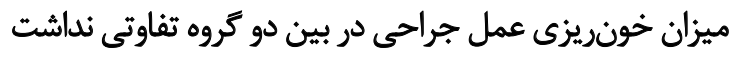

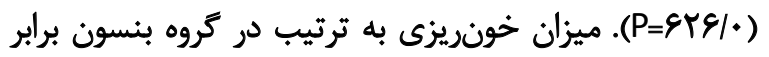
(INYIY/A (INIIY/F)

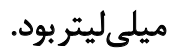

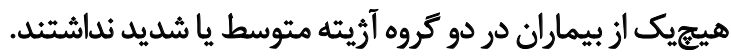

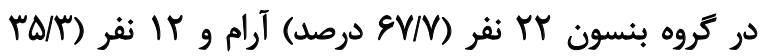

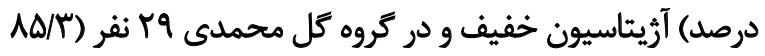

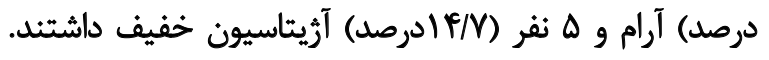

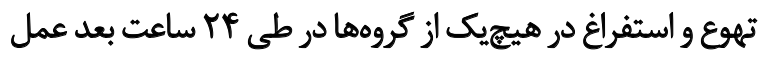
مشاهله نشل. ب هدف از اين مطالعه تعيين تأثير آرامسازى بنسون و رايحه

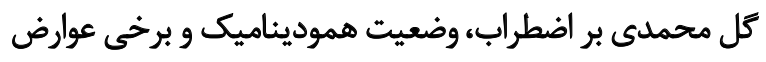

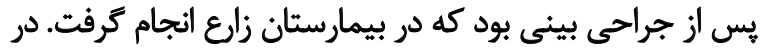

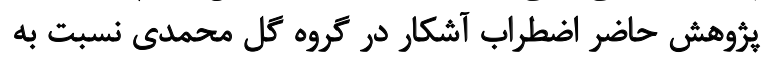

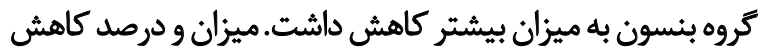

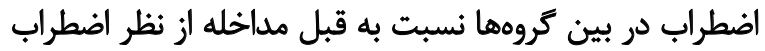

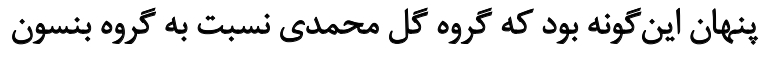

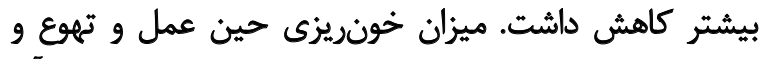

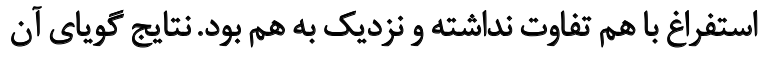


جدول r. مقايسه ميانكين فشار خون سيستول، دياستول و نبض بيماران در مرحله قبل و بعد از مداخله و ريكاورى به تفكيك در دو كروه كل محمدى و بنسون

\begin{tabular}{|c|c|c|c|c|}
\hline \multirow{2}{*}{ "سطح معنى دارى } & \multicolumn{2}{|c|}{ ميانكين土|نحراف معيار } & & \multirow{2}{*}{ كروهها متغير ها } \\
\hline & كل محمدى & بنسون & & \\
\hline $.1 \cdot p$ & $\Lambda 1 / N \pm N T$ & $v e / q \pm 1 \cdot / \Delta$ & قبل مداخله & \\
\hline .1 .08 & $8 \Delta / \& \pm \& / N$ & $V \cdot / f \pm V / \&$ & بعد معاخله & نبض (تعلداد در دقيقه) \\
\hline $.1 . .+$ & $V / R \pm V / \Delta$ & $V Q / P \pm N E$ & ريكاورى & \\
\hline.$/ 9 \Delta \Delta$ & $\pi r \cdot / \Delta \pm 9 / 9$ & $1 Y \cdot / 8 \pm 11 / 8$ & قبل مداخله & \\
\hline $.1 . .1$ & $1 \cdot \Delta / F \pm N F$ & $\|F / \gamma \pm\| / F$ & بعل معاخله & فُشار خون سيستوليك (ميلىمتر \\
\hline.$/ m$. & $M r+/ \pm N Y$ & $\mid r T / Y \pm 1$. & ريكاورى & \\
\hline . IATD & $V Q / Y \pm N \Delta$ & $1 \cdot / 8 \pm 9 / V$ & قبل مداخله & \\
\hline 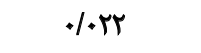 & $\varepsilon q / \Lambda \pm V / \Delta$ & $V * / v \pm q / \Delta$ & بعد ملاخله & فشار خون دياستوليك (ميلىمتر جيوه) \\
\hline.$/ 14 q$ & $V q / e \pm \Delta / f$ & $A T / T \pm N / F$ & ريكاورى & \\
\hline
\end{tabular}

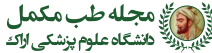

*t-test

مطالعه ما با تحقيق ميرزايى و همكاران همسو بود به كونهاي

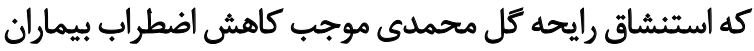

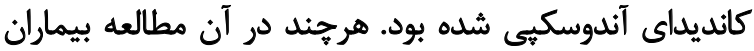

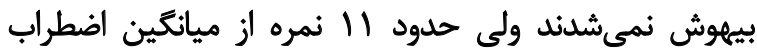

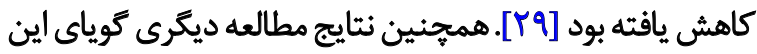

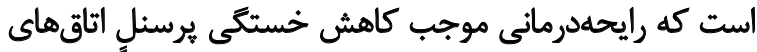

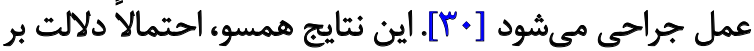

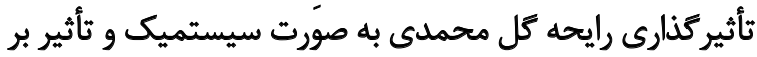

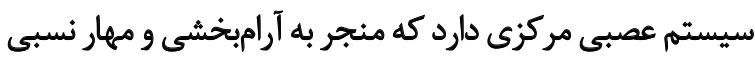

الكتروفيزيولوريك مورد بررسى قرار كرفت و به اين نتيجه رسيدند

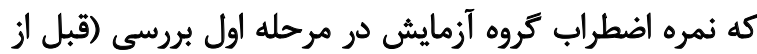

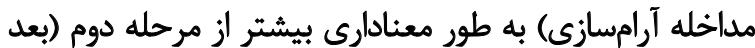

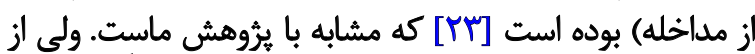

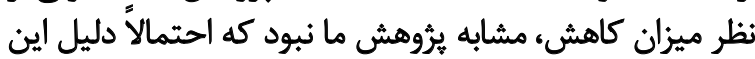

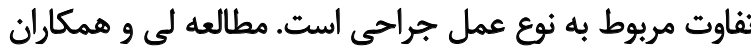

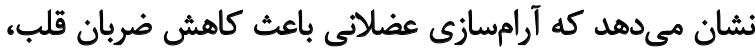

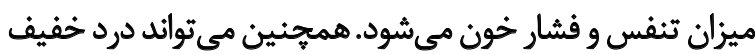
تا متوسط را كاهش دهد [Y [Y] كه همسو با مطالعه ماست. نتايج

جدول با. مقايسه ميانكين و درصد تغييرات اضطراب آشكار و ينهان قبل و بعد از مداخله در دو كروه

\begin{tabular}{|c|c|c|c|c|}
\hline \multirow{2}{*}{ "سطح معنى دارى } & \multicolumn{2}{|c|}{ ميانكين |نحرواف معيار } & \multirow{2}{*}{ مراحل } & \multirow{2}{*}{ مثغير } \\
\hline & ئروه كّل هحمدي & كئروه بنسون & & \\
\hline$\cdot(\cdot \Delta)$ & $F N F \pm \Delta / q$ & $r T / V \pm \lambda$ & قبل مداخله & \\
\hline.$/ 998$ & $r \Delta / 1 \pm \varphi / v$ & $\Pi \varphi / \Lambda \pm \Delta / \varphi$ & & اضطراب آشكار \\
\hline \multirow[t]{2}{*}{-} & $+1+1$ & $.1+1$ & Paired $t$ test & سطح معنى دارى \\
\hline & $\mid r / \Psi(T V / \Delta)$ & $N q(Y+/ F)$ & & نمره (درصد كاهش) \\
\hline.$/ 998$ & $F E / V \pm S / F$ & $r \& / V \pm \& / T$ & قبل مداخله & \\
\hline H.P & $m+\Delta / 1$ & $r v \pm r / \lambda$ & بعد مداخله & "שصراب (بهل \\
\hline$=$ & $.1 . .1$ & .10 .1 & Paired t test & سطح معنى دارى \\
\hline- & $I T / Y(T V / T)$ & $q / V(r+/ \Lambda)$ & & نمره (درصد كاهش) \\
\hline
\end{tabular}




$$
\text { مطالعات بيشترى رامىطلبد. }
$$

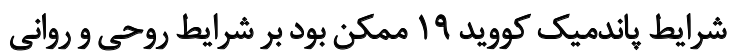

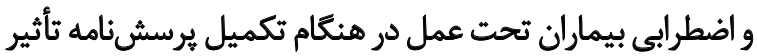

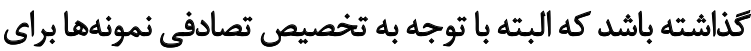

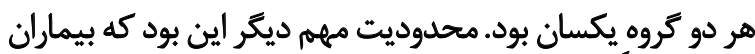

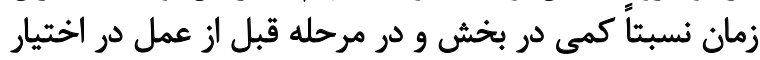

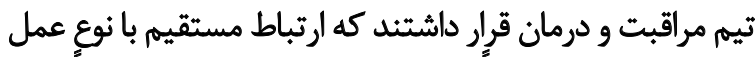

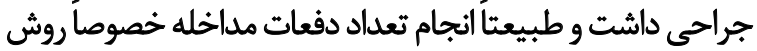

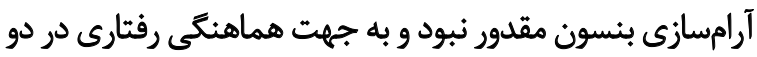

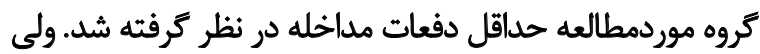

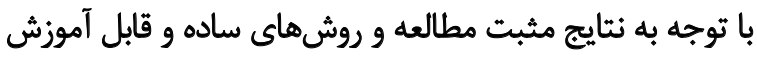

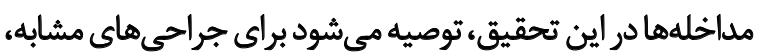

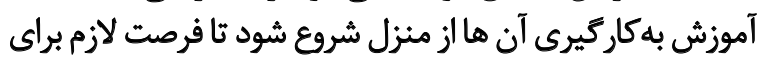
دفعات مناسب مداخله شكل تيردا

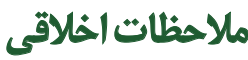 \\ يبيووى از اصول الخلاق ثيثوهشي}

اين مطالعه يس إزاخذ مجوز از كميته اخلاق دريثروهش دانشعاه

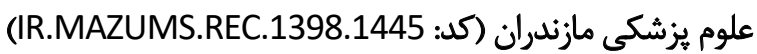

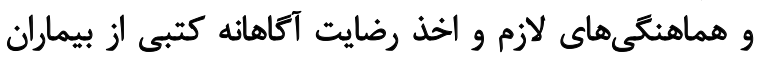

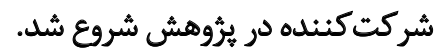

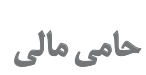

اين مقاله بخشى از هاياننامه كارشناسى ارشد نويسنده اول،

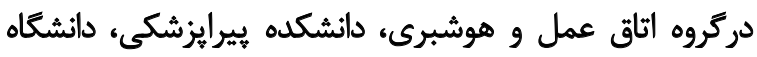

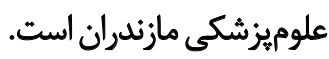

$$
\text { مشاركت نويسندكان }
$$

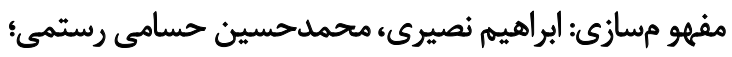

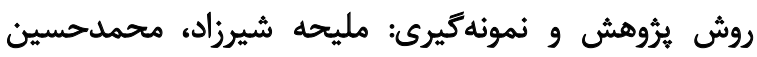

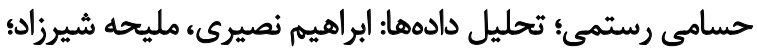
نكارش متن و بازبينى: تمام نويسندكان.

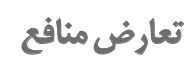

بنابر اظهار نويسندكان، اين مقاله تعارض منافع ندارد.

$$
\text { تشكر و قدرداني }
$$

از معاونت تحقيقات و فناورى دانشكاه علوميزشكى مازندران،

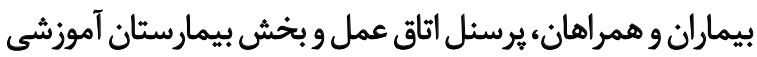

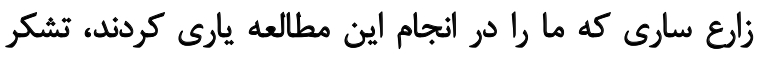
مى كنيهم.

$$
\text { اضطراب و خستكى مي فود. }
$$

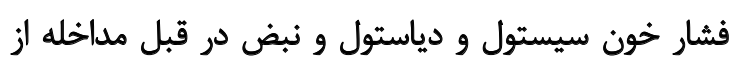

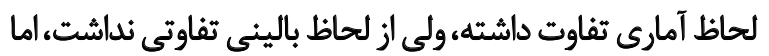

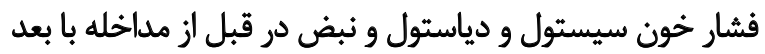

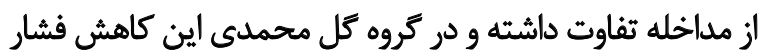

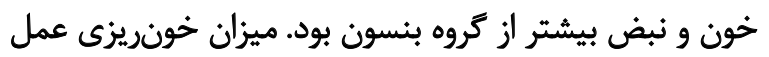
جراحى در بين دو كروه تفاوتى نداشت.

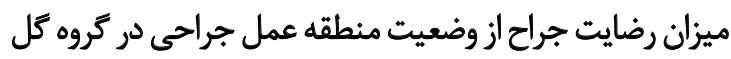

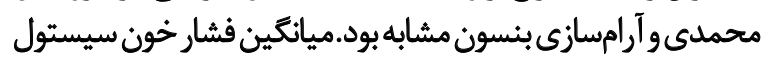

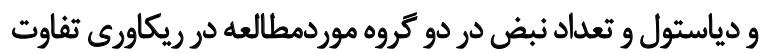

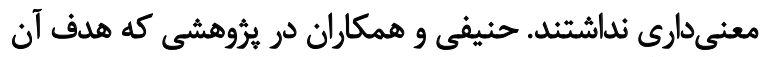

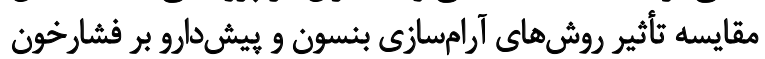

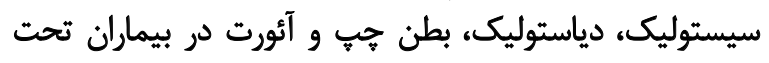

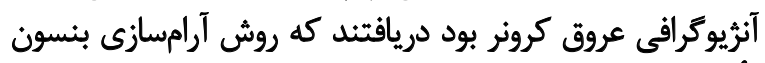

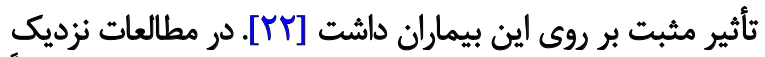

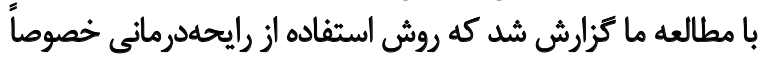

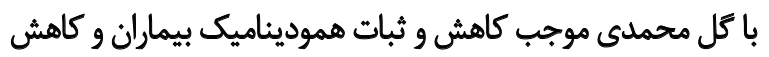

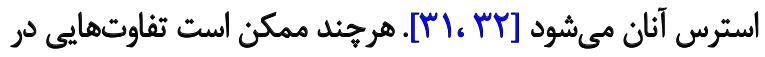

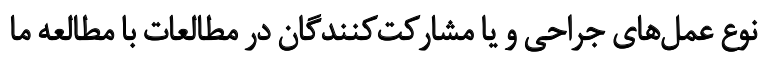

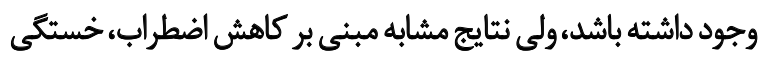

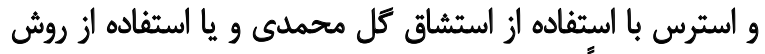

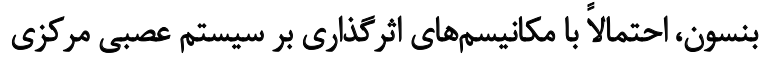

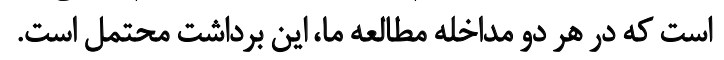

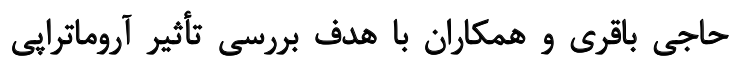

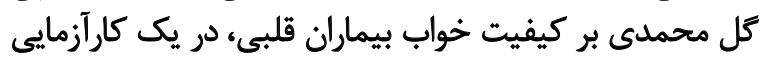

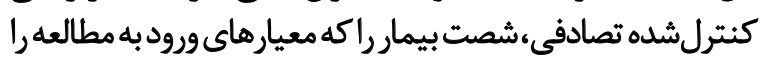

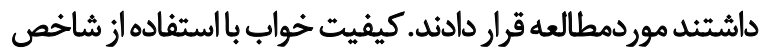

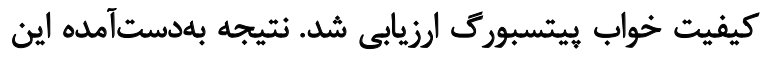

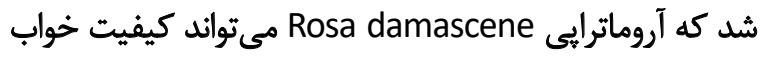

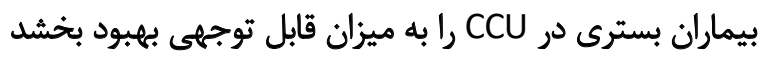

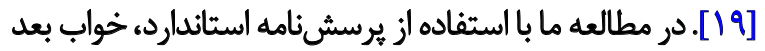

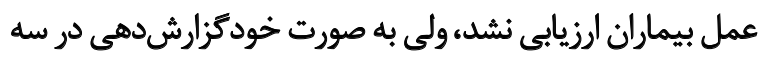

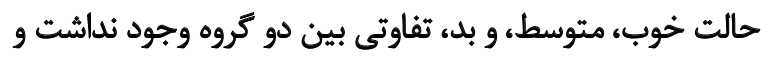
هر دو روش احساس بهترى از خواب را ايجاد كردند.

\section{نتيجلمَيرى}

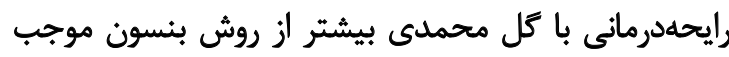

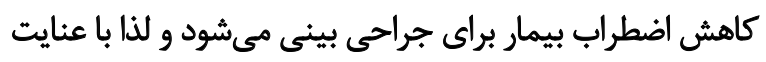

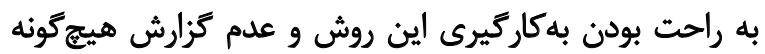

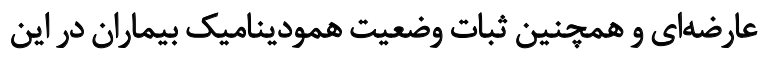

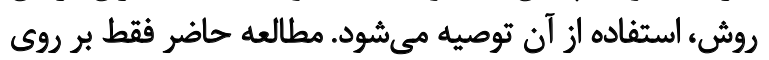

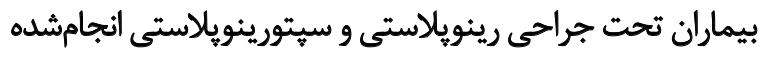

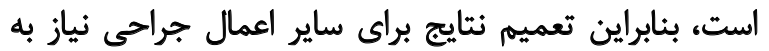




\section{References}

[1] Mohammadshahi M, Pourreza A, Orojlo PH, Mahmoodi M, Akbari F. Rhinoplasty as a medicalized phenomenon: A 25-center survey on quality of life before and after cosmetic rhinoplasty. Aesthetic Plastic Surgery. 2014; 38(4):615-9. [DOI:10.1007/s00266-014-0323-5] [PMID]

[2] Neaman KC, Boettcher AK, Do VH, Mulder C, Baca M, Renucci JD, et al. Cosmetic rhinoplasty: Revision rates revisited. Aesthetic Surgery Journal. 2013; 33(1):31-7. [DOI:10.1177/1090820X12469221]] [PMID]

[3] Shauly O, Calvert J, Stevens G, Rohrich R, Villanueva N, Gould DJ. Assessment of wellbeing and anxiety-related disorders in those seeking rhinoplasty: A crowdsourcing-based study. Plastic Reconstructive Surgery Global Open. 2020; 8(4):e2737. [DOI:10.1097/ GOX.0000000000002737] [PMID] [PMCID]

[4] Dagli R, Avcu M, Metin M, Kiymaz S, Ciftci H. The effects of aromatherapy using rose oil (Rosa damascena Mill.) on preoperative anxiety: A prospective randomized clinical trial. European Journal of Integrative Medicine. 2019; 26:37-42. [DOI:10.1016/j.eujim.2019.01.006]

[5] Zemła AJ, Nowicka-Sauer K, Jarmoszewicz K, Wera K, Batkiewicz S, Pietrzykowska M. Measures of preoperative anxiety. Anaesthesiology Intensive Therapy. 2019; 51(1):64-9. [DOI:10.5603/AIT.2019.0013] [PMID]

[6] Pereira L, Figueiredo-Braga M, Carvalho IP. Preoperative anxiety in ambulatory surgery: The impact of an empathic patient-centered approach on psychological and clinical outcomes. Patient Education Counseling. 2016; 99(5):733-8. [DOI:10.1016/j.pec.2015.11.016] [PMID]

[7] Dymond S. Overcoming avoidance in anxiety disorders: The contributions of Pavlovian and operant avoidance extinction methods. Neuroscience \& Biobehavioral Reviews. 2019; 98:61-70. [DOI:10.1016/j.neubiorev.2019.01.007]

[8] Mahfozi A, Hasani Mian M. The effect of midazolam in reducing anxity of surgical patients. Scientific Journal Of Forenisic Medicine. 2000; 6(19):20-3. https://www.sid.ir/en/Journal/ViewPaper.aspx?ID=26449

[9] Najafi Z, Taghadosi M, Sharifi K, Farrokhian A, Tagharrobi Z. The effects of inhalation aromatherapy on anxiety in patients with myocardial infarction: A randomized clinical trial. Iranian Red Crescent Medical Journal. 2014; 16(8): e15485. [DOI:10.5812/ircmj.15485] [PMCID] [PMID]

[10] Uğraş GA, Yıldırım G, Yüksel S, Öztürkçü Y, Kuzdere M, Öztekin SD. The effect of different types of music on patients' preoperative anxiety: A randomized controlled trial. Complementary Therapies in Clinical Practice. 2018; 31:158-63. [DOI:10.1016/j.ctcp.2018.02.012] [PMID]

[11] Ibrahim A, Koyuncu G, Koyuncu N, Suzer NE, Cakir OD, Karcioglu $O$. The effect of Benson relaxation method on anxiety in the emergency care. Medicine. 2019; 98(21):e15452. [DOI:10.1097/ MD.0000000000015452] [PMID] [PMCID]

[12] Seifi L, Najafi Ghezeljeh T, Haghani H. [The effects of benson relaxation technique and nature sound's on anxiety in patients with heart failure (Persian)]. Nursing And Midwifery Journal. 2017; 15(2):147-58. http:// unmf.umsu.ac.ir/article-1-3036-en.html

[13] Mogharab M, Ayoubzadeh K, Sharif-zadeh GR. Effect of peppermint aromatherapy on the level of anxiety in patients undergoing colonoscopy. Medical-Surgical Nursing Journal. 2017; 6(1):e67861. https://sites. kowsarpub.com/msnj/articles/67861.htm

[14] Gholami M, Teymouri F, Farsi Z, Rajai N. [The effect of aromatherapy with essential oil of damascena on the anxiety of nurses working in the emergency department of the selected hospital of AJA University of Medical Sciences (Persian)]. Military Caring Sciences. 2019; 5(4):282-91. https://www.sid.ir/fa/journal/ViewPaper.aspx?ID=481301
[15] Kane A. Childbirth and aromatherapy. International Journal of Childbirth Education. 1997; 12(1):14-5. https://www.proquest.com/openview/9f 37380519f86241adf681a6cf9007b/1?pq-origsite=gscholar\&cbl=32235

[16] Heidari MR, Nateq M, Ebadi A. Aromatherapy from the perspective of traditional Iranian medicine and modern medicine. Journal of Islamic and Iranian Traditional Medicine. 2017; 8(2):173-82. http://jiitm.ir/ article-1-855-en.html

[17] Bastani F, Samady Kia P, Haghani H. The effect of inhalation aromatherapy with damask rose (rosa damascena) on the pain of elderly after knee arthroplasty. Journal of Client-Centered Nursing Care. 2017 3(2):153-160. http://jccnc.iums.ac.ir/article-1-137-fa.htm

[18] Bikmoradi A, Harorani M, Roshanaei G, Moradkhani S, Falahinia $\mathrm{GH}$. [The effect of inhalation aromatherapy with damask rose (Rosa damascena) essence on the pain intensity after dressing in patients with burns: A clinical randomized trial (Persian)]. Iranian Journal of Nursing Midwifery Research. 2016; 21(3):247-54. [DOI:10.4103/17359066.180380] [PMID] [PMCID]

[19] Hajibagheri A, Babaii A, Adib-Hajbaghery M. Effect of Rosa damascene aromatherapy on sleep quality in cardiac patients: a randomized controlled trial. Complementary Therapies in Clinical Practice. 2014 20(3):159-63. [DOI:10.1016/j.ctcp.2014.05.001] [PMID]

[20] Keyhanmehr AS, Movahhed M, Sahranavard S, Gachkar L, Hamdieh M Afsharpaiman $\mathrm{S}$, et al. The effect of aromatherapy with Rosa damascena essential oil on sleep quality in children. Research Journal of Pharmacognosy. 2018; 5(1):41-6. https://eprints.bmsu.ac.ir/2958/1/The\%20 Effect\%20of\%20Aromatherapy\%20with\%20Rosa\%20damascena\%20 Essential\%200il\%20on\%20Sleep\%20Quality\%20in\%20Children.pdf

[21] Teimouri F, Pishgooie SA, Malmir M, Rajai N. The effect of Benson relaxation on physiological criteria in patients undergoing coronary artery bypass graft surgery. Complementary Medicine Journal. 2019 9(3):3813-23. [DOI:10.32598/cmja.9.3.3812]

[22] Hanifi N, Ahmadi F, Memarian R, Khani M. [The effect of benson relaxation technique and premedication on systolic, diastolic, LV \& Aorta Pressure in Patients under Coronary Artery Angiography (Persian)]. Razi Journal of Medical Sciences. 2005; 12(46):287-94. http://rjms.iums. ac.ir/article-1-459-en.html

[23] Jafari H, Baghaei Lake M, Sedghisabet M, Kazemnejadleili E. [Benson Muscle Relaxation effect on Patients Anxiety Undertake Diagnostic and Treatment within Electrophysiological Interventions (Persian)]. Journal of Holistic Nursing Midwifery. 2015; 25(4):37-44. http://hnmj.gums. ac.ir/article-1-577-en.html

[24] Abolhasani S. [Investigation of the effect of sensory stimulations on sleep deprivation symptoms in patients hospitalized in coronary care unit (Persian)]. Koomesh. 2005; 7(1-2):71-6. https://koomeshjournal.semums. ac.ir/article-1-116-en.pdf

[25] Abolhasani S. [Investigation of the effect of sensory stimulations on sleep deprivation symptoms in patients hospitalized in coronary care unit (Persian)]. Koomesh. 2006; 7(1):71-6. http://koomeshjournal.semums.ac.ir/article-1-116-fa.htm

[26] Rigi F, Bazdar P, Salehi Ardabili S, Naseri M, Feizi A. [The effect of foot reflexology on anxiety in patients with coronary artery bypass surgery referred to Seyed-Al-Shohada teaching hospital, Urmia, 2012 (Persian)] The Journal of Urmia Nursing Midwifery Faculty. 2013; 11(8):578-83. http://unmf.umsu.ac.ir/article-1-1514-en.html

[27] Dehghani A, Kalami L, Seyyedhejazi M, Nasery RA, Marjani K. The efficacy of oral clonidine and oral midazolam in prevention of emergence ag itation in post-operative pediatric. Majallah pizishki-i Danishgah-i Ulum-i Pizishki va Khadamat-i Bihdashti-i Darmani-i Tabriz. 2014; 35(6):44-51. https://mj.tbzmed.ac.ir/Article/8919 
[28] Hashemy S, Zakerimoghadam M. [Comparative study of the effect of muscle relaxation and music therapy on anxiety level in patients waiting for cardiac catheterization (Persian)]. Iranian Journal of Cardiovascular Nursing. 2013; 1(4):22-30. http://journal.icns.org.ir/article-1-102-en. html

[29] Lee EJ, Bhattacharya J, Sohn C, Verres R. Monochord sounds and progressive muscle relaxation reduce anxiety and improve relaxation during chemotherapy: A pilot EEG study. Complementary Therapies in Medicine. 2012; 20(6):409-16. [DOI:10.1016/j.ctim.2012.07.002] [PMID]

[30] Mirzaee Jirdehi M, Monfared A, Mansour Ghanaei F, Kazemnezhad Leili E. Damask rose aromatherapy and the anxiety of endoscopic candidate patients: A clinical trial. Journal of Holistic Nursing and Midwifery. 2021; 31 (3):203-11. [DOI:10.32598/jhnm.31.3.2126]

[31] Akhule OZ, Nasiri E, Lotfi M, Mahmoodi A, Akbari H. [The effect of concomitant exercise and inhalation of lavender fragrance on surgical technologists' fatigue severity (Persian)]. Journal of Health and Safety at Work. 2021; 11(1):26-39. https://jhsw.tums.ac.ir/files/site1/user_ files_0ab0c0/eng/sayeh-A-10-74-73-bc18e48.pdf

[32] Ahmed Mohammed ELmetwaly A, Gaad El Moula Shaaban E, Mahmoud Hafez Mohamed E. Benson relaxation technique: Reducing pain intensity, anxiety level and improving sleep quality among patients undergoing thoracic surgery. Egyptian Journal of Health Care. 2020; 11(4):602-14. [DOI:10.21608/ejhc.2020.160893]

[33] Bikmoradi A, Roshanaei G, Moradkhani S, Fatahi A. Impact of inhalation aromatherapy with damask rose on anxiety of patients undergoing coronary angiography: A single-blind randomized controlled clinical trial avicenna. Journal of Nursing and Midwifery Care. 2020; 28(2):93-102. [DOI:10.1515/jcim-2020-0415.] [PMID] 